\title{
MORPHOLOGICAL PROPERTIES OF ELLIPTICAL GALAXIES
}

\author{
S. Djorgovski \\ Harvard-Smithsonian Center for Astrophysics \\ 60 Garden St. \\ Cambridge, MA 02138, USA.
}

\begin{abstract}
In the poster as presented at the meeting, I described global morphological properties of elliptical galaxies, based on the data from a CCD surface photometry survey of $\sim 200$ ellipticals and $\sim 50$ S0's (Djorgovski 1985). In this brief summary, I emphasize two points: (1) there is a very weak and very noisy trend of radial shape with luminosity, in the sense that more luminous galaxies are less concentrated, and (2) there is no preference for low-luminosity ellipticals to show boxy isophotes, and they differ in that respect from the bulges.
\end{abstract}

There is a wide variety of shapes of surface brightness profiles, which implies any formula or model which do not contain at least one shape parameter (e.g., the $r^{1 / 4}$ law, Binney, or Jaffe models) cannot describe satisfactorly all (or any?) elliptical galaxies. There are no obvious systematics in this variety, except for the very slight trend with luminosity, illustrated in Figure 1. The trend is in the sense that more luminous galaxies are less concentrated, or have shallower surface brightness profiles; for example, it is well known that cD's behave in that way, with respect to lower luminosity ellipticals. Schombert (1986) argued that this trend is well defined and that one can construct a "standard" surface brightness profile at any given luminosity. I agree that there is a trend, but it is by far too noisy, and at any given luminosity there is too much diversity in profile shapes.

The galaxies show also a wide variety of ellipticity profiles. There is a tendency toward positive ellipticity gradients, but there are exceptions. The gradients usually flatten into a constant ellipticity at some radius, and sometimes reverse after that. The isophotal twists are common, but not too large, typically a few degrees per decade in radius. The ellipticities, ellipticity gradients and isophotal twist rates are not mutually correlated, and none of them is correlated with luminosity, radial shape of light distribution, or any of the kinematical quantities.

Davies et al. (1983) demonstrated that the low luminosity ellipticals and bulges are similar in their dynamical properties. They suggested that, in analogy with boxy or peanut-shaped bulges, the low luminosity ellipticals may show a higher incidence of boxy isophotes. Figure 2 demonstrates that this is not the case: there is no trend of "boxiness" (or, for that matter, "diskiness") with luminosity. The small elliptical and bulges are not equivalent in all of their properties. 


\section{REFERENCES}

Davies, R., et al. 1983, Astrophys. J. 266, 41. (DEFIS)

Djorgovski, S. 1985, Ph.D. Thesis, University of California, Berkeley.

Schombert, J. 1986, Astrophys. J. Suppl. Ser. 60, 603.

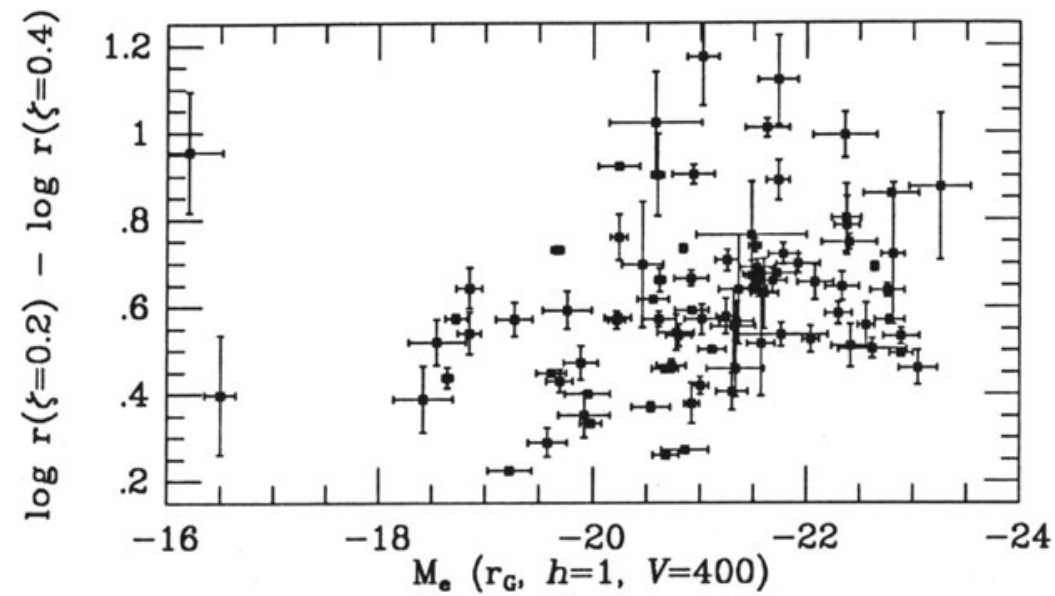

Figure 1: Ratio of two fiducial radii (in a logarithmic form), plotted vs. the luminosity. The more luminous galaxies tend to have shallower light profiles, and thus larger radial ratios. The very discrepant point at $M_{e}=-16.2$ is $\mathrm{M} 32$, which may be a post-core-collapse galaxy. A similar trend is also seen when a magnitude difference in two fiducial apertures is plotted against the luminosity.

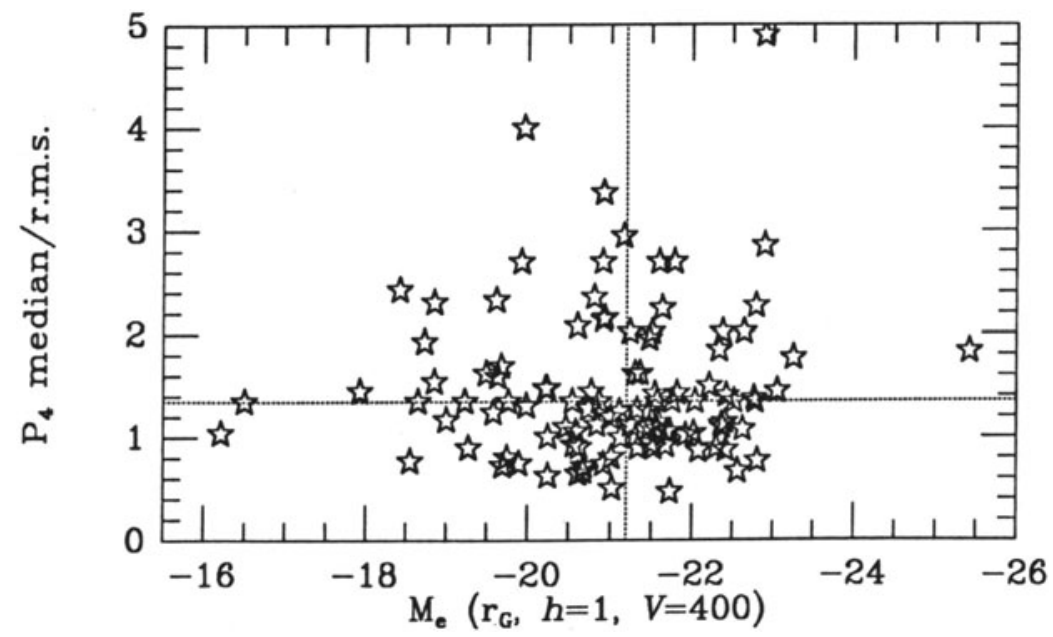

Figure 2: A parameter indicating the mean "boxines" of a galaxy (residual Fourier 4-wave amplitude, renormalized by the error-bar) plotted $v s$. the luminosity. The dotted lines indicate sample medians in both coordinates. There is no trend with luminosity. A Virgocentric infall model with $V_{\text {inf }}=400 \mathrm{~km} \mathrm{~s}^{-1}$, and $h=1$ were used in computing the absolute luminosities. 